\title{
Fluctuations in partitioning systems with few degrees of freedom
}

\author{
L. Cerino \\ Dipartimento di Fisica, Università di Roma Sapienza, p.le A. Moro 2, 00185 Roma, Italy \\ G. Gradenigo \\ CEA/DSM-CNRS/URA 2306, CEA Saclay, F-91191 Gif-sur-Yvette Cedex, France and \\ Univ. Paris Sud; CNRS; LPTMS, UMR 8626, Orsay 91405 France
}

\begin{abstract}
A. Sarracino
ISC-CNR and Dipartimento di Fisica, Università Sapienza, p.le A. Moro 2, 00185 Roma, Italy, Kavli Institute for Theoretical Physics China, CAS, Beijing 100190, China and

Laboratoire de Physique Théorique de la Matière Condensée, CNRS UMR 7600, case courrier 121, Université Paris 6, 4 Place Jussieu, 75255 Paris Cedex

D. Villamaina

Laboratoire de Physique Théorique de l'ENS and Institut de Physique

Théorique Philippe Meyer, 24 rue Lhomond 75005 Paris - France

\author{
A. Vulpiani \\ Dipartimento di Fisica, Università Sapienza and ISC-CNR, p.le A. Moro 2, 00185 Roma, Italy and \\ Kavli Institute for Theoretical Physics China, CAS, Beijing 100190, China
}

\begin{abstract}
We study the behavior of a moving wall in contact with a particle gas and subjected to an external force. We compare the fluctuations of the system observed in the microcanonical and canonical ensembles, at varying the number of particles. Static and dynamic correlations signal significant differences between the two ensembles. Furthermore, velocity-velocity correlations of the moving wall present a complex two-time relaxation which cannot be reproduced by a standard Langevin-like description. Quite remarkably, increasing the number of gas particles in an elongated geometry, we find a typical timescale, related to the interaction between the partitioning wall and the particles, which grows macroscopically.
\end{abstract}

PACS numbers: $05.70-\mathrm{a}, 05.20 .-\mathrm{y}, 02.50 . \mathrm{Ey}$

\section{INTRODUCTION}

Macroscopic objects contain, at least, $N=O\left(10^{20}\right)$ particles, therefore in the mathematical modeling, one can safely assume $N \rightarrow \infty$ and study their asymptotic features (e.g. the thermodynamics limit). As consequence of such a huge value of $N$, up to few decades ago statistical mechanics has been devoted almost only to the study of systems with many degrees of freedom [1]. On the other hand the present day instrumentation allows us for the manipulation (and sometimes control) of small systems at micro, and even nano, scales; it is not necessary to emphasize the practical relevance of small systems [2, 3].

In order to deal with systems with a small number of particles, say $O\left(10^{2}\right)$ or less, we are forced to (re)consider in details some aspects of the statistical mechanics [4] which for macroscopic bodies are not very relevant. For instance in large systems the fluctuations are always relatively negligible (and apparently) irrelevant [5]. In a similar way, for macroscopic objects, there are not particular problems for the definition of temperature [6] neither significant differences using different statistical ensembles (e.g. microcanonical or canonical).

Among the physical systems relevant for the nanosciences we can mention the class of partitioning objects containing an extra degree of freedom (a wall) which separates the system into subsystems. A paradigmatic example is given by the adiabatic piston [7-11]: a system of $N$ particles of mass $m$ (e.g. an ideal gas) in a container of length $L$ and cross-section $A$, separated in two regions by a movable wall (the piston) of mass $M$. The walls of the container are supposed to be perfect insulators preventing any mass or heat exchanges with the exterior. Gas particles undergo purely elastic collisions with the piston and the walls, and the piston is constrained to move along one axis. If at initial time the temperatures $T_{L}, T_{R}$ and pressures $P_{L}, P_{R}$ in the left and right parts do not coincide, the system shows a rather rich phenomenology (depending on $M / m, N / L$ etc) in the approach to the mechanical and thermodynamic equilibrium.

A physical version of the adiabatic piston is a big Brownian particle sliding along a microtubule filled with particles [12]. The authors of ref [12] showed how the presence of the wall is able to induce, even in the equilibrium state, rather complex (and slow) dynamical behavior.

Our paper is devoted to the statistical mechanics of a system similar to a piston where particles are confined in a tube with a non fixed wall, on which an external force 
acts, see Fig. 1. The pressure on the piston due to the interaction with the gas particles on one side is balanced by the external force, so that the piston reaches a stationary state. We are interested in the study of piston fluctuations (of position and velocity) around the equilibrium state. In the case of non interacting particles it is possible to find in an exact way the equilibrium properties of the system both in microcanonical and canonical ensembles (this latter case is realized by putting a thermostat on the fixed wall, which thermalizes particles colliding with the wall). One obtains that, even in the limit $N \gg 1$, the fluctuations of the wall position are different in the canonical and microcanonical ensembles. As important consequence of such a difference, which holds also for the interacting particles, we have that the correlation function (of the velocity) $C(t)$ must be different in the two ensembles.

Numerical simulations show a non trivial behavior of $C(t)$ with a negative minimum around a characteristic time $\tau(N)$ increasing linearly with $N$. A comparison between the numerical results and an appropriate Langevin equation shows how even for large $N$ the presence of the wall has non trivial consequences which can have a role for an effective modeling of the system.

The paper is organized as follows: Section 2 describes the model in detail and presents the analytical results for the ideal gas case; in Section 3 we report the results of molecular dynamics simulations in the interacting case. Section 4 is devoted to the derivation of an effective Langevin equation for describing the dynamics of the piston, and, finally, in Section 5 some conclusions are drawn. Two Appendices provide details about the computations.

\section{THE MODEL}

We consider a two-dimensional system composed by a gas of $N$ point-like particles with mass $m$, positions $\mathbf{x}_{i}=\left\{x_{i}, y_{i}\right\}$ and momentum $\mathbf{p}_{i}$, with $i=1, \ldots, N$, contained in a rectangular box with one moving adiabatic wall of length $L$ (hereafter referred to as the "piston"). The position of the piston is denoted by $Y$ and its momentum and mass are $P$ and $M$, respectively (see Fig 1 for visual explanation). An external force $\mathbf{F}=-F \cdot \hat{y}$, directed along the horizontal axis $\hat{y}$, acts on the piston, which is also subject to the collisions with the particles. In the tubular geometry that we consider, in which the size of the sistem is increased anisotropically only along one direction when adding particles, the piston plays the role of a "partitioning" object with respect to the particle gas, namely its position determines the volume available for the gas. This system has been studied in [6] as an effective thermometer model. In the following the particleparticle and particle-piston interactions are described in a Hamiltonian (conservative) context and the piston can slide without dissipation along the $y$ axis. The case of dissipative interactions, inducing nonequilibrium behav-

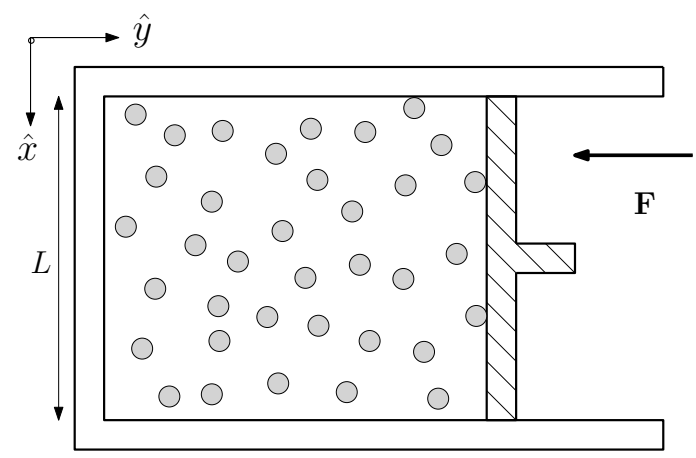

FIG. 1. Sketch of the piston model: a gas of particles is confined by a moving wall which is subjected to a constant external force.

iors, of similar systems have been studied for instance in 13 18.

We start by considering the case of a non-interacting gas, so that the Hamiltonian of the system reads

$$
\mathcal{H}=\sum_{i=1}^{N} \frac{\left|\mathbf{p}_{i}\right|^{2}}{2 m}+\frac{P^{2}}{2 M}+F Y,
$$

with geometrical constraints

$$
\left\{\begin{array}{l}
Y>0 \\
0<x_{i}<L \\
0<y_{i}<Y
\end{array}\right.
$$

We are interested in the study of the behavior of fluctuations at varying the number of gas particles, and, in particular, in the comparison between the microcanonical and canonical ensembles. As shown in the Appendix A, in the microcanonical ensemble the temperature of the system is related to the energy $E$ of the system by the relation

$$
k_{B} T=k_{B}^{2}\left(\frac{\partial \log \Sigma(E)}{\partial E}\right)^{-1}=\frac{E}{2 N+\frac{3}{2}},
$$

where

$$
\Sigma(E)=\int_{\mathcal{H}<E} d^{N} \mathbf{x} d^{N} \mathbf{p} d Y d P
$$

is the phase space volume and $k_{B}$ the Boltzmann's constant. The static properties of this system, average position $\langle Y\rangle$ and variance $\sigma_{Y}^{2}=\left\langle Y^{2}\right\rangle$, can be readily obtained (see Appendix A), yielding

$$
\begin{aligned}
\langle Y\rangle & =\frac{(N+1) k_{B} T}{F}, \\
\sigma_{Y}^{2} & =\frac{(N+1 / 2)(N+1)}{2 N+5 / 2}\left(\frac{k_{B} T}{F}\right)^{2} .
\end{aligned}
$$

Let us open a parenthesis on the definition of temperature. Eq. (3) is not the unique possibility, another way is via the formula

$$
k_{B} T^{\prime}=k_{B}^{2}\left(\frac{\partial \ln \omega(E)}{\partial E}\right)^{-1}
$$


where $\omega(E)=\frac{\partial \Sigma(E)}{\partial E}$. There are cases where $T$ and $T^{\prime}$ can be different and, in particular, $T^{\prime}$ can be negative, e.g. in the case of point vortex systems 19]. On the other hand, in a perfect gas it is easy to see that the two definitions are equivalent for $N \gg 1$ since $T-T^{\prime}=O\left(\frac{1}{N}\right)$ [20]; this result also holds for weakly interacting systems.

Analogous results can be obtained for the canonical case, where the system is in contact with a reservoir at temperature $T$. In this case, the energy of the system is

$$
E=-\frac{\partial \log Z(\beta)}{\partial \beta}=\left(2 N+\frac{2}{3}\right) k_{B} T,
$$

where

$$
Z(\beta)=\int d^{N} \mathbf{x} d^{N} \mathbf{p} d Y d P e^{-\beta \mathcal{H}}
$$

and $\beta=1 /\left(k_{B} T\right)$ is the inverse temperature. Average position and variance $\sigma_{Y}^{2}$ read (see Appendix A)

$$
\begin{aligned}
\langle Y\rangle & =\frac{(N+1) k_{B} T}{F}, \\
\sigma_{Y}^{2} & =(N+1)\left(\frac{k_{B} T}{F}\right)^{2} .
\end{aligned}
$$

In order to compare the results for static quantities $\langle Y\rangle$ and $\sigma_{Y}^{2}$ in the two ensembles, for each temperature $T$ in the canonical ensemble we consider the corresponding energy in the microcanonical, such that $T=E /\left(2 N k_{B}\right)$, in the limit of large number of particles $N \gg 1$. While the average position is always the same, from Eqs. (6) and (11) one observes that fluctuations differ by a factor $1 / 2$, also in the large $N$ limit. In the Table $\square$ we summarize these findings.

The equivalence of ensembles in the thermodynamic limit is expected only for average values, and not for fluctuations [4, 21]. Indeed, the observed "discrepancy" is explained by noting that the variance in the canonical ensemble can be expressed as the sum of two contributions, namely a term which corresponds to the variance of the piston in the microcanonical ensemble at fixed energy plus a term corresponding to energy fluctuations at fixed temperature:

$$
\sigma_{Y}^{2}(T)=\alpha \sigma_{E}^{2}(T)+\left.\sigma_{Y}^{2}(E)\right|_{E=\langle E\rangle_{\beta}},
$$

where $\alpha=1 /\left(4 F^{2}\right)+O(1 / N)$ and $\sigma_{E}^{2}=\left\langle\mathcal{H}^{2}\right\rangle-E^{2}$. Therefore, for $N \gg 1$, since $\sigma_{E}^{2}(T) \approx 2 N\left(k_{B} T\right)^{2}$, one has $\sigma_{Y}^{2}(T)=\left.2 \sigma_{Y}^{2}(E)\right|_{E=2 N k_{B} T}$.

Let us open a short digression on terminology. With the term "canonical ensemble" we mean the system with Hamiltonian in Eq. (11) and Eq. (2) (in the following we will include also the interactions among the particles) interacting with a thermal bath at temperature $T$. Noting that the pressure is nothing but $F / L$, one can then say that we are dealing with an ensemble at fixed temperature and fixed pressure for the system without the terms $F X$ and $P^{2} /(2 M)$ in the Hamiltonian [21]. In a similar
Canonical|Microcanonical

\begin{tabular}{c|c|c}
\hline Temperature: & $T$ & $\frac{E}{2 N k_{B}}$ \\
$\langle Y\rangle$ & $\frac{N k_{B} T}{F}$ & $\frac{E}{2 F}=\frac{N k_{B} T}{F}$ \\
$\sigma_{Y}^{2}$ & $\frac{N\left(k_{B} T\right)^{2}}{F^{2}}$ & $\frac{E^{2}}{8 N F^{2}}=\frac{N\left(k_{B} T\right)^{2}}{2 F^{2}}$
\end{tabular}

TABLE I. Comparison of average position and variance in the microcanonical and canonical ensembles.

way our microcanonical ensemble correspond to an ensemble with fixed enthalpy for the system without the terms $F X$ and $P^{2} /(2 M)$ in the Hamiltonian. We prefer the terms canonical and microcanonical because they put the dynamical variables describing the wall on the same level of those for the particles. Let us note that the mass of the piston is important for the dynamical properties.

The above results on the fluctuations immediately produce two important consequences on the dynamical correlations in the two ensembles. First, notice that the finite value of the variance $\sigma_{Y}^{2}$ in both cases for finite $N$ implies that the diffusion coefficient $D$ of the piston is zero, implying that the piston remains confined. Second, the difference in the static fluctuations have repercussions on the shape of the velocity-velocity fluctuations in the canonical and microcanonical ensemble. Let us note that

$$
\sigma_{Y}^{2}=\left\langle(Y-\langle Y\rangle)^{2}\right\rangle=\int_{0}^{\infty} \int_{0}^{\infty}\left\langle V\left(t^{\prime}\right) V\left(t^{\prime \prime}\right)\right\rangle d t^{\prime} d t^{\prime \prime}
$$

where $V(t)$ is the velocity of the piston. Since $\sigma_{Y}^{2}$ are different in the canonical and microcanonical ensembles also the correlation $\langle V(t) V(0)\rangle$ must be different. These issues will be addressed in the next section, in the case of interacting gas.

Exactly the same considerations about the difference of fluctuations in the canonical and microcanonical ensembles hold in the case that a different termodynamic limit is considered, in wich the size of the piston is increased isotropically. In this case, in order to have that for each value of $N$ the shape of the gas compartement is isotropic, namely $\langle Y\rangle=L$, and that the density $\rho=N / L^{2}$ and the pressure $p=F / L$ are constant, we need the scaling $F \sim \sqrt{N}$ for the force acting on the piston. If we insert such scaling for $F$ in the equations Eq. 66111), we find that increasing isotropically the size of the compartiment, at variance with the tubular geometry, the mean square dispacement $\sigma_{Y}^{2}$ of the partitioning wall becomes asimptotically costant for increasing $N$ in the two ensembles. On the contrary the factor 2 by which canonical and microcanical fluctuations differ remains the same. The comparison between the two different thermodynamic limits tell us on one hand that the result on the difference in canonical and microcanonical fluctuations is robust and on the other hand allows us to point out the peculiarities of the tubular geometry. 


\section{NUMERICAL SIMULATIONS FOR THE INTERACTING CASE}

In order to understand whether the previous results are peculiar to the non-interacting case, and to study a more realistic case, we perform molecular dynamics simulations of the system with an interacting particle gas. We consider a repulsive interaction potential $V(\mathbf{r})$ for soft disks, with cut-off $r_{c}$

$$
V(\mathbf{r})=\left\{\begin{array}{cc}
V_{0}\left[\left(\frac{r_{0}}{r}\right)^{12}-\left(\frac{r_{0}}{r_{c}}\right)^{12}+12\left(\frac{r_{0}}{r_{c}}\right)^{12}\left(\frac{r}{r_{c}}-1\right)\right] & \text { for } r<r_{c} \\
0 \quad \text { for } r>r_{c}, &
\end{array}\right.
$$

where $r=|\mathbf{r}|$ is the distance between particles, $V_{0}$ is the potential intensity and $r_{0}$ is the average interaction range. The same potential also describes the interaction of particles with walls. In the simulations of the canonical ensemble the coupling with the reservoir at temperature $T$ is implemented in the following way. We consider that the side of the box opposite to the piston acts as a thermostat, so that when a particle enters the interaction region with the wall, namely its distance from the wall is smaller than $r_{0}$, the velocity is changed along the $y$ axis according to the Maxwellian distribution $p\left(v_{y}\right) \propto v_{y} \exp \left(-v_{y}^{2} / 2 m k_{B} T\right)$, for $v_{y}>0$ [22]. The study of the system upon varying $N$ is performed by retaining a tubular geometry, namely keeping the length $L$ and the force $F$ constant and letting the equilibrium position $\langle Y\rangle$ increase accordingly, so that the gas density remains fixed. The results here described are not related to a specific interacion. Ideed, we also studied the case of a stronger interaction potential $V(r) \sim r^{-64}$, which at low density reproduces the behavior of hard-disk statistics [23], finding analogous results.

We start the numerical study of this interacting case by checking the validity of the relation (3). In Figure 2 we plot the temperature $T$ as a function of the energy $E$ in the microcanonical and canonical ensembles. The temperature is computed as $k_{B} T=M\left\langle V^{2}\right\rangle$ whereas energy is $E=\langle\mathcal{H}\rangle$. As expected, the theoretical relation (3) derived in the non-interacting system is valid at high temperatures, where interactions become negligible. In Figure 3 we report the average values of the piston position and its variance in the two ensembles. Notice that also in this case the analytical predictions (5) and (10) hold in the high energy (or temperature) regions.

It is interesting the fact that also in the interacting case the factor $1 / 2$ between the $\sigma_{Y}^{2}$ in the canonical and microcanonical is still present (see Fig. 4).

Interesting behaviors are also found for the dynamical properties of this system. Indeed, differences in the fluctuations between microcanonical and canonical are evident from the study of correlation functions. In particular, in Figure 5 we compare the behavior of the normalized velocity autocorrelation function of the piston, $C(t)=\langle V(t) V(0)\rangle /\langle V(0) V(0)\rangle$, for different values of

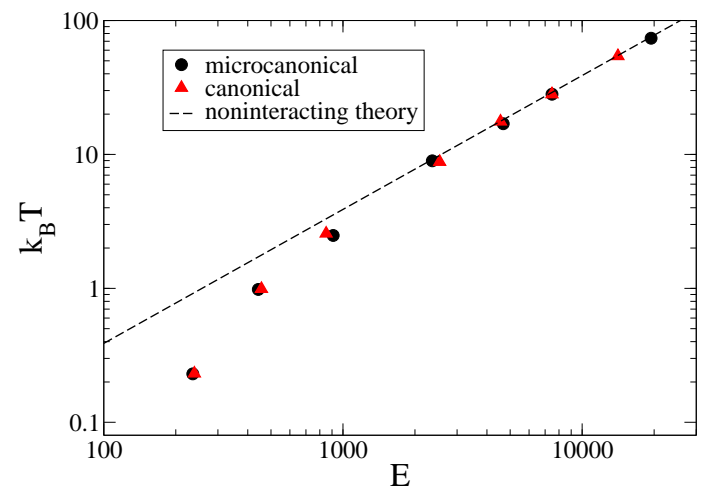

FIG. 2. (color online) The temperature $k_{B} T$ (in the microcanonical ensemble is $\left.M\left\langle V^{2}\right\rangle\right)$ is plotted as a function of energy $E$ (in the canonical ensemble $E=\langle\mathcal{H}\rangle$ ) for $N=128$. The dashed line represents the theoretical result for noninteracting particles $k_{B} T=E /(2 N+3 / 2)$, which is expected to hold for high temperatures. Other parameters in the simulations are $L=10, F=10, m=1$ and $M=128$.
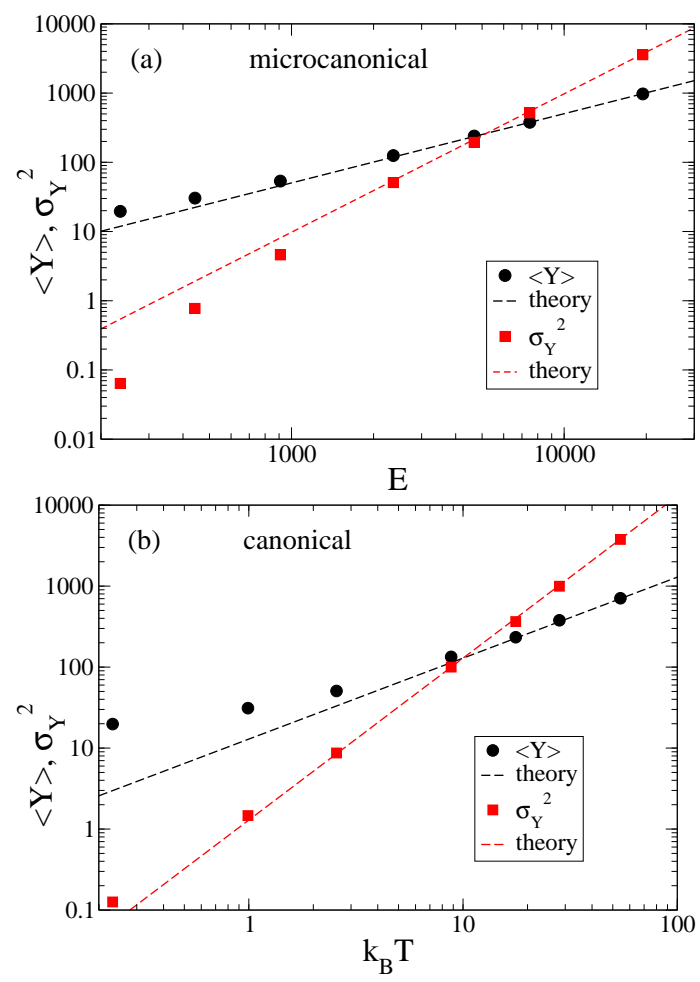

FIG. 3. (color online) Panel (a): The average position $\langle Y\rangle$ and variance $\sigma_{Y}^{2}$ are plotted as a function of energy $E$ in the microcanonical ensemble with $N=128$. Dashed lines represent the theoretical results for the non-interacting gas: $\langle Y\rangle=(N+1) E /(2 N+3 / 2)$ and $\sigma_{Y}^{2}=(N+1)(N+1 / 2) /[(2 N+$ $\left.5 / 2)(2 N+3 / 2)^{2}\right](E / F)^{2}$. Panel (b): Same quantities as a function of $k_{B} T$ in the canonical ensemble. Theoretical results for the non-interacting gas are: $\left.\langle Y\rangle=(N+1) k_{B} T / F\right)$ and $\sigma_{Y}^{2}=(N+1)\left(k_{B} T\right)^{2} / F^{2}$. Other parameters in the simulations are $L=10, F=10, m=1$ and $M=128$.

$N$. First, one clearly observes that, as expected from 


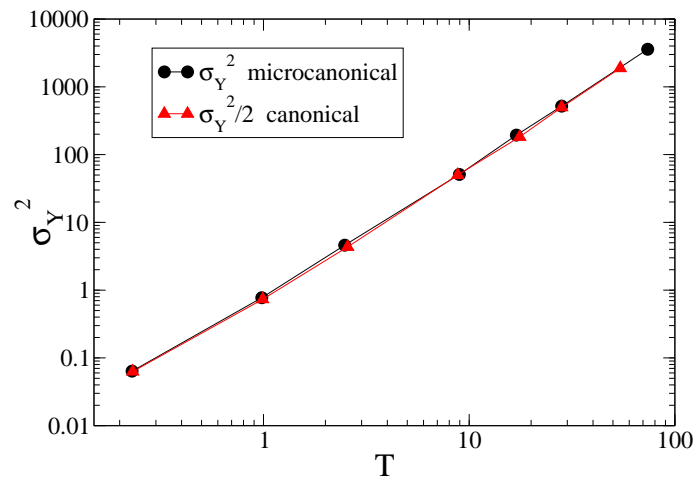

FIG. 4. (color online) The variance $\sigma_{Y}^{2}$ is plotted as a function of $k_{B} T$ in the canonical and microcanonical ensembles (in the latter case we consider simulations at constant energy and the temperature is obtained from $k_{B} T=M\left\langle V^{2}\right\rangle_{E}$ ) for $N=128$. Other parameters in the simulations are $L=10, F=10$, $m=1$ and $M=128$.

the static results, fluctuations are larger in the canonical ensemble, namely the system is less correlated than in the microcanonical. Moreover, let us notice the nontrivial shape of $C(t)$. For small $N$ one has a damped oscillatory relaxation, while, increasing $N$, a peculiar behavior emerges: after a first stage of relaxation, governed by a simple exponential decay, at later times a negative bump occurs, signaling the presence of another timescale in the system. This negative contribution to the correlation is necessary for the vanishing of the diffusion constant: $\int_{0}^{\infty} C(t) d t$ must be zero.

From the above results for $C(t)$, a two-time scenario emerges. We have the time $\tau_{0}$, characterizing the first exponential decay, empirically defined as the time necessary to cross the zero axis for the first time. In addition, we have the time $\tau(N)$ where the negative bump occurs. The first decay of the velocity correlation function $C(t)$ saturates upon increasing the number of particles and so the time $\tau_{0}$ tends to a constant value, independent of $N$ (see panel (a) of Figure 6] where $\tau_{0}$ is plotted as a function of $N$ in semilog scale, both for the microcanonical and the canonical ensembles). On the other hand, we find that the second timescale $\tau$ depends linearly on $N$, as it is shown in panel (b) of Figure 6, where $C(t)$ is plotted as a function of $t / N$. In the inset we also plot $\tau(N)$ as a function of $N$ in log-log scale for the canonical ensemble, showing the linear increasing with $N$ (analogous results are observed for the microcanonical ensemble).

As discussed in the next section, such a peculiar behavior, induced by the presence of the partitioning piston, cannot be easily described by a standard Langevin-like approach.

\section{LANGEVIN EQUATION}

In the limit of $N$ and $M$ very large, the relaxation times of the piston and of the gas particles are well separated,
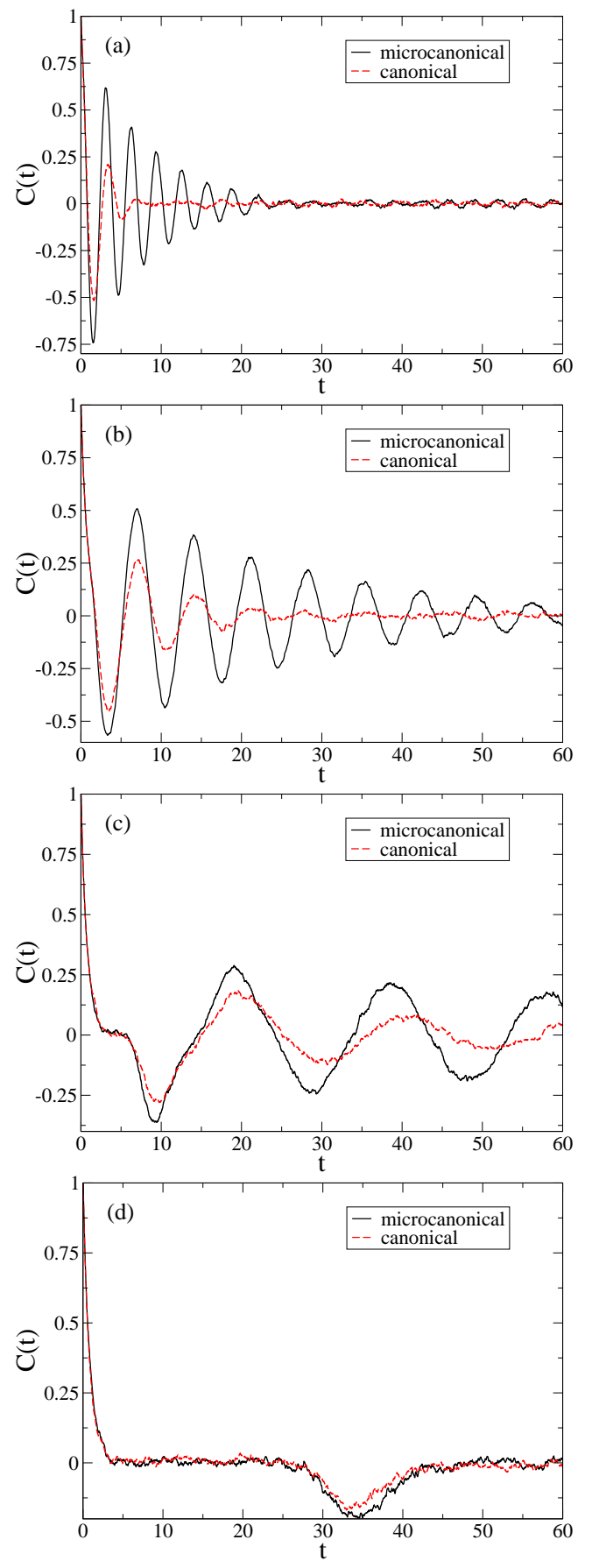

FIG. 5. (color online) Velocity autocorrelation functions of the piston in the microcanonical and canonical ensembles, for $N=16$, panel (a), $N=64$, panel (b), $N=256$, panel (c) and $N=1024$, panel (d). Other parameters are $L=30, F=150$, $T=10, m=1$ and $M=50$.

and one may consider the gas particles weakly perturbed by the presence of the piston.

Within this strong assumption, the gas distribution is fixed and independent of the motion of the piston, and the dynamics can be described by a master equation for the probability density function $P(V, Y, t)$ from the veloc- 

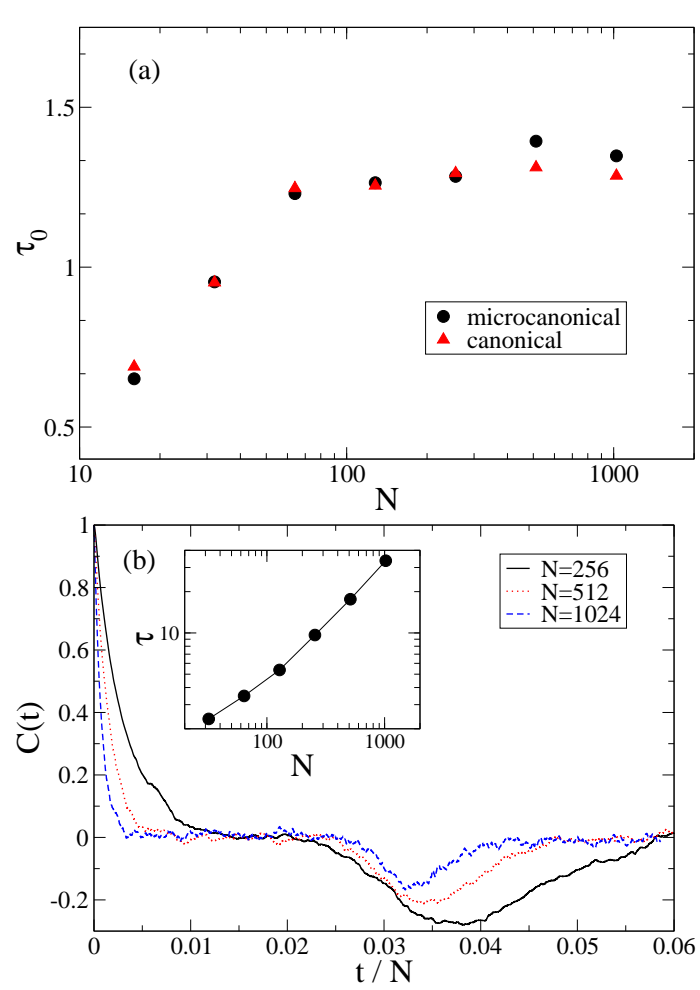

FIG. 6. (color online) Panel (a): first relaxation time $\tau_{0}$ of the piston velocity correlation for different values of $N$, in the microcanonical (black dote) and canonical (red squares) ensemble with parameters $M=50, F=150, L=30$ and $k_{B} T=10$. Notice that the first relaxation saturates for large $N$, and the time $\tau_{0}$ reaches a constant value, both in the canonical and microcanonical ensembles. Panel (b): velocity correlation functions as a function of time rescaled by $N$ in the canonical ensemble with same parameters. In the inset the time $\tau$ shows a linear dependence on $N$, for large $N$.

ity $V$ of the piston at position $Y$ at time $t$. In particular, for the first moment of this distribution, it is possible to write down the following equation (for the details refer to the Appendix $\overline{\mathrm{VB}}$ ):

$$
\frac{d\langle V\rangle}{d t}=\left\langle F_{\text {coll }}(Y, V)\right\rangle
$$

Then, the fluctuations around the equilibrium position ( $Y \simeq Y_{e q}$ and $V \simeq 0$ ) are described by expanding up to the first order the right hand side of Eq. (15), obtaining

$$
\frac{d V(t)}{d t}=-k_{N} y-\gamma V+\xi(t),
$$

where the displacement $y \equiv Y-Y_{e q}$ has been introduced. The parameters $k_{N}$ and $\gamma$ can be calculated by means of kinetic theory, and their explicit expressions are written in Eq. (46) of Appendix $\mathrm{VB}$. One must notice that in Eq. (16) a noise term $\xi(t)$ has been added, whose expression cannot be directly derived from the Eq. (15) for the mean velocity. Actually, the correlation of the noise term can be determined by exploiting equipartition theorem valid for equilibrium dynamics. By requiring Maxwellian statistics for the stationary $P(V)$, it is well known that $\xi(t)$ must be white noise with variance

$$
\left\langle\xi(t) \xi\left(t^{\prime}\right)\right\rangle=2 \gamma T \delta\left(t-t^{\prime}\right) .
$$

From the linearity of Eq. (16) it is possible to calculate the autocorrelation of velocity, obtaining:

$$
\langle V(t) V(0)\rangle=\frac{T}{M} e^{-\frac{\gamma t}{2}}\left[\cosh \left(\frac{\Delta}{2} t\right)-\frac{\gamma \sinh \left(\frac{\Delta}{2} t\right)}{\Delta}\right]
$$

where we intruduced the parameter $\Delta=\sqrt{\gamma^{2}-4 k_{N}}$, which rules the passage between underdamped and overdamped regime. More specifically, if $\frac{N m}{m+M}>\frac{\pi}{2}$, the system is overdamped, else the system is underdamped.

Making a comparison between Eq. (18) and the numerical experiments presented in Fig. 5 it appears evident that the Langevin equation is able to capture, for $N$ large, only the small time relaxation $\tau_{0} \simeq \gamma^{-1}$, while is unable to detect the oscillation of $\langle V(t) V(0)\rangle$, that appears for times $\tau(N) \sim N$. We report in Fig. 7 the explicit comparison between the Langevin approximation (black curve) and the piston velocity correlation (red curve) in the non-interacting case. The same mismatch between analytical prediction and numerical results is observebd also for interacting particles. The oscillations presented by $\langle V(t) V(0)\rangle$ are related with the interplay mechanism between the moving wall and a collective mode of the gas particles, that make the assuption of Markovianity to fail. We note how this phenomenon is quite general and it is present also in the case of noninteracting gas particles. In order to verify this point, one can analyze a natural collective variable of the gas, i.e. the center of mass velocity $v_{c m}(t) \equiv \frac{1}{N} \sum v_{i}(t)$. In the simpler case of a non-interacting gas confined in a fixed volume, the autocorrelation $\left\langle v_{c m}(t) v_{c m}(0)\right\rangle$ would be trivially equal to the one of a single particle in the gas. On the contrary this is not true anymore with the presence of the piston, since the different particles of the gas strongly correlates each other via the mutual interaction piston/border. The time scale of this process is very close to $\tau(N)$, as it can be observed in Fig. 7 Such a time scale is completely hidden if one consider only the single particle autocorrelation $\left\langle v_{i}(t) v_{i}(0)\right\rangle$.

\section{CONCLUSIONS}

In the present work we have shown, with analitical calculations in the ideal gas case and with simulations for interacting particles, that the fluctuations in the canonical and microcanonical ensembles [27] show relevant differences when a partitioning object, like a moving wall, is introduced. The relevant points that we have highlighted are the following. First, we have shown that the interaction with the partitioning object induces nontrivial correlations among the particles even in the ideal gas 


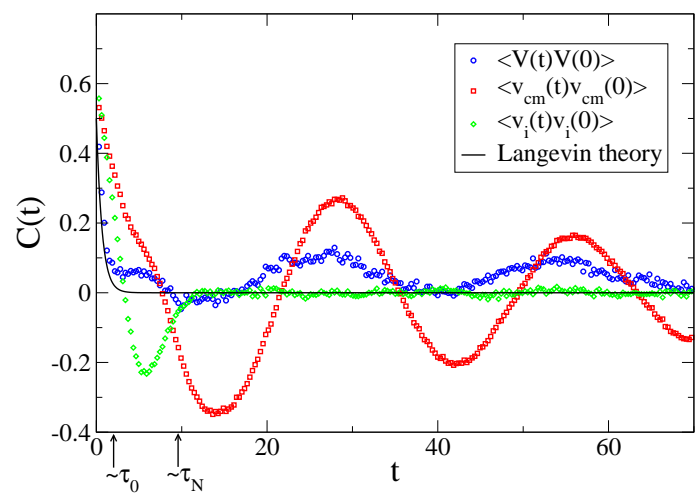

FIG. 7. (color online) Autocorrelation of different observables in the case of a piston with non-interacting particles (canonical ensemble) measured in numerical simulations and Langevin approximation for the piston velocity correlation (black line). It is possible to observe how the oscillation in the autocorrelation of the piston velocity $\langle V(t) V(0)\rangle$ (blue circles) are in phase with the one of the center of mass of the gas particles $\left\langle v_{c m}(t) v_{c m}(0)\right\rangle$ (red squares). With green diamonds is represented the autocorrelation of a single particle velocity $\left\langle v_{i}(t) v_{i}(0)\right\rangle$. All the correlations are normalized to one for $t=0$. Value of the parameters: $F=150, T=10$, $M=50, N=500$.

approximation, see Fig. [7in Sec.IV] irrespectively of the ensemble, canonical or microcanonical, where the dynamics is studied. Then, we have shown that the Langevin approach to the dynamics of the piston captures only partially the physics of the system. The Langevin equation, correctly predicts only the fast time scale, namely $\tau_{0} \sim \gamma^{-1}$, but fails completely to catch the slower one, which grows linearly with the number of particles in the partitioned system, $\tau(N) \sim N$. This second time scale is produced by non-trivial correlations among the velocity of the gas particles and the one of the piston which are present, quite remarkably, also in the case of noninteracting particles, as shown in Fig. 7 .

We recall that the macroscopic growth of $\tau(N)$ is related to the particular tubular geometry of the problem, where the size of the gas compartment is increased only in one direction. Notwithstanding the different behavior of the largest timescale, the factor 2 of difference between canonical and microcanonical fluctuations of the partitioning object $\sigma_{Y}^{2}$, is independent from how the thermodynamic limit is taken, how is clear from Eq. (6) and Eq. (11).

We can therefore conclude that partitioning geometries with a single macroscopic degree of freedom which is effectively coupled to the motion of all the microscopic costituents of the system represent an eligible framework to study the dynamical properties of small systems.

\section{APPENDIX A}

\section{A. Microcanonical}

In the microcanonical ensemble at energy $E$, the invariant measure is non-zero only on the ipersurface of constant energy $S_{E}$. If $\mathcal{M}$ is a subset of $S_{E}$ and $d \sigma$ is the infinitesimal surface element

$$
\mathbb{P}\left(x \in \mathcal{M} \subseteq S_{E}\right)=\int_{\mathcal{M}} \frac{d \sigma}{\omega(E)} \frac{1}{|\nabla \mathcal{H}|},
$$

where $\omega(E)=\partial \Sigma(E) / \partial E$. In order to derive the expression of the temperature of the system as a function of the energy, we must compute $\Sigma(E)$. This quantity is given by

$$
\begin{aligned}
\Sigma(E) & =\int_{\mathcal{H}<E} d^{N} x d^{N} y d Y d^{N} \mathbf{p} d P \\
& =L^{N} \int_{\sum_{i}\left|\mathbf{p}_{i}\right|^{2} / 2 m+P^{2} / 2 M+F Y<E} d^{N} y d Y d^{N} \mathbf{p} d P .
\end{aligned}
$$

Recalling that the volume of a $D$-dimensional sphere of radius $R$ is $V(R)=\int_{\sum_{i} x_{i}^{2}<R^{2}} d^{D} x=\frac{\pi^{\frac{D}{2}}}{\Gamma\left(\frac{D}{2}+1\right)} R^{D}$, where $\Gamma(x)$ is the Euler Gamma, from Eq. (20) we obtain

$$
\begin{aligned}
\Sigma(E) & =(2 m)^{N} \sqrt{2 M} L^{N} \frac{\pi^{N+\frac{1}{2}}}{\Gamma\left(N+\frac{3}{2}\right)} \\
& \times \int_{0}^{Y} d^{N} y \int_{0}^{E / F} d Y(E-F Y)^{N+\frac{1}{2}} \\
& =(2 m)^{N} \frac{\sqrt{2 M}}{F}\left(\frac{L}{F}\right)^{N} \frac{\pi^{N+\frac{1}{2}}}{\Gamma\left(N+\frac{3}{2}\right)} E^{2 N+\frac{3}{2}} \\
& \times \int_{0}^{1} d x x^{N}(1-x)^{N+\frac{1}{2}}
\end{aligned}
$$

and, eventually,

$$
\Sigma(E)=(2 m)^{N} \frac{\sqrt{2 M} L^{N}}{F^{N+1}} \pi^{N+\frac{1}{2}} \frac{\Gamma(N+1)}{\Gamma\left(2 N+\frac{5}{2}\right)} E^{2 N+\frac{3}{2}},
$$

and

$$
\omega(E)=(2 m)^{N} \frac{\sqrt{2 M} L^{N}}{F^{N+1}} \pi^{N+\frac{1}{2}} \frac{\Gamma(N+1)}{\Gamma\left(2 N+\frac{3}{2}\right)} E^{2 N+\frac{1}{2}}
$$

Now we can compute the temperature of the system using the relation $S=k_{B} \ln \Sigma(E)$, namely

$$
k_{B} T=k_{B}\left(\frac{\partial S}{\partial E}\right)^{-1}=\frac{\Sigma(E)}{\omega(E)}=\frac{E}{2 N+\frac{3}{2}} .
$$

Using alternative definitions of $S$, e.g. $S=k_{B} \ln \omega(E)$ or $S=k_{B} \ln \Gamma_{\Delta E}(E)$, where $\Gamma_{\Delta E}(E)=\Sigma(E+\Delta E)-$ $\Sigma(E) \simeq \omega(E) \Delta E$ where $\Delta E$ is the tolerance on $E$, for $N \gg 1$ one has negligible differences [20]. 
We are interested in the probability density function of the position of the piston $Y$. Observing that for a generic phase space function $A(\mathbf{X})$ in the microcanonical ensemble one has [25]:

$$
\rho_{A}(a)=\frac{1}{\omega(E)} \frac{\partial}{\partial E} \mathcal{I}(E, a),
$$

where

$$
\mathcal{I}(E, a)=\int_{\mathcal{H}<E} \delta(A(\mathbf{x})-a) d \mathbf{x},
$$

putting $A(\mathbf{X})=Y$ one readily obtains

$$
\begin{aligned}
I(E, Y=\tilde{Y}) & =\int_{H<E} d Y d^{N} x d^{N} y d^{N} \mathbf{p}_{i} d p \delta(Y-\tilde{Y}) \\
& =(2 m)^{N} \sqrt{2 M} L^{N} \frac{\pi^{N+\frac{1}{2}}}{\Gamma\left(N+\frac{3}{2}\right)} \tilde{Y}^{N}(E-F \tilde{Y})^{N+\frac{1}{2}},
\end{aligned}
$$

for $0<\tilde{Y}<E / F$; therefore

$$
\begin{aligned}
\rho_{E}(Y) & =\frac{1}{\omega(E)} \frac{\partial I}{\partial E}=\frac{\Gamma\left(2 N+\frac{3}{2}\right)}{\Gamma\left(N+\frac{1}{2}\right) \Gamma(N+1)} \\
& \times \frac{F}{E}\left(\frac{F Y}{E}\right)^{N}\left(1-\frac{F Y}{E}\right)^{N-\frac{1}{2}} .
\end{aligned}
$$

From the above result, we obtain

$$
\langle Y\rangle=\frac{(N+1) k_{B} T}{F}
$$

and

$$
\sigma_{Y}^{2}=\frac{\left(N+\frac{1}{2}\right)(N+1)}{2 N+\frac{5}{2}}\left(\frac{k_{B} T}{F}\right)^{2},
$$

where, in the two last equations, we used Eq. (24) to express $\langle Y\rangle$ and $\sigma_{Y}^{2}$ as functions of $T$ instead of $E$.

\section{B. Canonical}

In the canonical ensemble at constant temperature $T$ with $\beta=1 /\left(k_{B} T\right)$, the partition function of the system is given by

$$
\begin{aligned}
Z & =\int d^{N} x d^{N} y d Y d^{N} \mathbf{p} d P e^{-\beta \mathcal{H}}= \\
& =\left(\frac{2}{\pi}\right)^{N+\frac{1}{2}} N ! m^{N} \sqrt{M} \beta^{-\left(2 N+\frac{3}{2}\right)} F^{-(N+1)} .
\end{aligned}
$$

We can easily compute the mean energy of the system

$$
E=\langle\mathcal{H}\rangle=-\frac{\partial \ln Z}{\partial \beta}=\left(2 N+\frac{3}{2}\right) k_{B} T .
$$

Now we want to find the probability distribution function of the position of the piston $Y$ : starting from

$$
\rho_{\beta}\left(Y,\left\{y_{i}\right\}\right)=\frac{e^{-\beta F Y}}{\int d Y d^{N} y e^{-\beta F Y}} \prod_{i} \Theta\left(Y-y_{i}\right),
$$

and integrating over all the $y_{i}$, one obtains

$$
\rho_{\beta}(Y)=\frac{Y^{N} e^{-\beta F Y}}{\int d Y Y^{N} e^{-\beta F Y}} .
$$

The mean value of this distribution is

$$
\langle Y\rangle=\frac{k_{B} T(N+1)}{F}
$$

whereas its variance is

$$
\sigma_{Y}^{2}=\frac{(N+1)\left(k_{B} T\right)^{2}}{F^{2}} .
$$

\section{APPENDIX B}

In this appendix, we detail the derivation of the Langevin equation for the motion of the piston, following elementary kinetic theory. The basic idea is to estimate the average force exerted by the gas particles which collide with the piston, by calculating the average momentum exchanged in the collisions. The following approach dates back to Smoluchowski 24] and it has been used to write a Langevin equation for colloidal particles [26]. For the variable $y=Y-Y_{e q}$ we will derive a stochastic equation

$$
M \frac{d^{2} y}{d t^{2}}=F_{a v}(y, \dot{y})+C \eta
$$

where $F_{a v}(y, \dot{y})$ is the average force acting on the piston in the position $Y_{e q}+y$ and velocity $\dot{y}, \eta$ is a white noise and the constant $C$ can be fixed a posteriori from the condition $M\left\langle\dot{y}^{2}\right\rangle=k_{B} T$.

Consider the gas at equilibrium, and focus on the collision of the piston, characterized by its mass $M$ and precollisional velocity $V$, and a particle of the gas, which are characterized by $m$ and $\mathbf{v}$, respectively. The collision rule is

$V^{\prime}=V+\frac{2 m}{m+M}\left(v_{y}-V\right) \quad v_{y}^{\prime}=v_{y}-\frac{2 M}{m+M}\left(v_{y}-V\right)$

where the primed quantity are postcollisional velocities, and $v_{y}$ is the $y$-component of $\mathbf{v}$. The rate of such collisions can be obtained by considering the equivalent problem of a piston, at rest, hit by a flux of particles moving at relative velocity $V \hat{\mathbf{y}}-\mathbf{v}$. The rate is then determined by counting the number of point-like particles hitting the unit surface in the infinitesimal time interval $d t$. This number corresponds to the particles contained in a rectangle of infinitesimal base length $\delta x$ and height $\left(v_{y}-V\right) \Theta\left(v_{y}-V\right) d t$. The step function $\Theta(s)$ selects the condition for having a collision. Setting $v=v_{y}$, the mean 
force exerted by the particles of the gas on the piston is

$$
\begin{aligned}
& F_{\text {coll }}(Y, V)=\left\langle M \frac{\Delta V}{d t}\right\rangle \\
= & M \int_{-\infty}^{\infty} d v \int_{0}^{L} d x \rho\left(x, Y-r_{0}^{\prime}\right) \\
\times & \phi(v)\left(V^{\prime}-V\right)(v-V) \Theta(v-V) \\
= & \frac{2 m M}{m+M} \\
\times & \int_{-\infty}^{\infty} d v \int_{0}^{L} d x \rho\left(x, Y-r_{0}^{\prime}\right) \phi(v) \Theta(v-V)(v-V)^{2}
\end{aligned}
$$

where $\phi(v)$ is the equilibrium distribution of velocities of the gas, i.e. $\phi(v)=\sqrt{\frac{m}{2 \pi k_{B} T}} e^{-\frac{m v^{2}}{2 k_{B} T}}$ and $\rho(x, Y)$ is the spatial density of particles in the proximity of the piston. At equilibrium, this density is uniform on all the available volume and, therefore, depends on the position of the piston $Y$. Carrying on the integration on the spatial coordinates, we obtain

$$
F_{\text {coll }}(Y, V)=\frac{2 m M}{m+M} \lambda \int_{V}^{\infty} d v(v-V)^{2} \phi(v)
$$

where $\lambda=\frac{N}{Y}$. We note that the equilibrium properties of the gas used in the derivation of this equation don't depend on the choice of the ensemble. Of course, $F_{a v}(y, \dot{y})$ is nothing but $F_{\text {coll }}-F$.

In order to decouple the motion of the piston from the one of the gas molecules it's necessary to assume that $M \gg m$ and that, moreover, $V$ is always small if compared to the thermal velocity of the particles $v_{m}=\sqrt{\frac{2 k_{B} T}{m}}$ : the expansion of the integral in Eq. (40) in powers of $\sqrt{\frac{m}{M}}$, will give the viscous drag force appearing in the Langevin equation of motion. Defining $g=\sqrt{\frac{m}{2 k_{B} T}}(v-V)$ and expanding perturbatively $\phi(v)$ as a function of $g$

$$
\begin{aligned}
e^{-\frac{m}{2 k_{B} T} v^{2}} & =e^{-\left(g+\sqrt{\frac{m}{2 k_{B} T}} V\right)^{2}} \simeq e^{-g^{2}-\sqrt{\frac{2 m}{k_{B} T}} g V} \\
& \simeq e^{-g^{2}}\left(1-\sqrt{\frac{2 m}{k_{B} T}} g V\right)
\end{aligned}
$$

we can compute the integral, performing the change of variables $v \rightarrow g$

$\frac{2 k_{B} T}{m \sqrt{\pi}} \int_{0}^{\infty} g^{2} e^{-g^{2}}\left(1-\sqrt{\frac{2 m}{k_{B} T}} g V\right) d g=\frac{k_{B} T}{2 m}-\sqrt{\frac{2 k_{B} T}{\pi m}} V$

namely

$$
F_{\text {coll }}=\frac{N}{Y}\left[\frac{M}{m+M} k_{B} T-2 \frac{M}{m+M} \sqrt{\frac{2 m k_{B} T}{\pi}} V\right]
$$

Expanding the previous expression at the first order in $y$ and $V$ around the equilibrium position of the piston $Y_{e q}$, defined by the condition $F=F_{\text {coll }}$ and $V=0$, we obtain a linear Langevin equation. The equilibrium conditions are

$$
\frac{M}{m+M} k_{B} T \frac{N}{Y_{e q}}=F \text { and } V_{e q}=0
$$

and therefore

$$
Y_{e q}=\frac{N M k_{B} T}{F(m+M)} .
$$

The Langevin equation has the shape

$$
\frac{d^{2} y}{d t^{2}}=-k_{N} y-\gamma v+\frac{C}{M} \eta
$$

where

$$
\gamma=\frac{2 F}{M} \sqrt{\frac{2 m}{\pi k_{B} T}} \text { and } k_{N}=\frac{F^{2}(m+M)}{M^{2} N k_{B} T} .
$$

It is easy to compute the correlation function

$$
\langle V(t) V(0)\rangle=\frac{k_{B} T}{M} e^{-\frac{\gamma}{2}}\left[\cosh \left(\frac{\Delta}{2} t\right)-\frac{\gamma}{\Delta} \sinh \left(\frac{\Delta}{2} t\right)\right],
$$

where $\Delta=\sqrt{\gamma^{2}-4 k_{N}}$. Let us note that for any finite $N$ (i.e. $k_{N} \neq 0$ ) one has $\int_{0}^{\infty}\langle V(t) V(0)\rangle d t=0$.

\section{ACKNOWLEDGMENTS}

We thank M. Falcioni and A. Puglisi for useful discussions. The work of AS is supported by the Granular Chaos project, funded by the Italian MIUR under the grant number RIBD08Z9JE.
[1] P. Castiglione, M. Falcioni, A. Lesne and A. Vulpiani, Chaos and Coarse Graining in Statistical Mechanics (Cambridge University Press, 2008).

[2] C. Bustamante, J. Liphardt and F. Ritort F, Physics Today 58, 43 (2005).

[3] M. L. Simpson and P. T. Cummings, ACS Nano 4, 2425 (2011).
[4] J. L. Lebowitz, J. K. Percus and L. Verlet, Phys. Rev. 153, 250 (1967).

[5] U. Marini Bettolo Marconi, A. Puglisi, L. Rondoni, A. Vulpiani, Phys. Rep. 461, 111 (2008).

[6] M. Falcioni, A. Puglisi, A. Sarracino, D. Villamaina, A. Vulpiani, Am. J. Phys. 79, 777 (2011).

[7] B. Crosignani, P. Di Porto and M. Segev, Am. J. Phys. 
64, 610 (1996).

[8] C. Gruber and J. Piasecki, Physica A 268, 412 (1999).

[9] C. Gruber, S. Pache and A. Lesne, J. Stat. Phys. 112, 1177 (2003).

[10] C. Van den Broeck, R. Kawai and P. Meurs, Phys. Rev. Lett. 93, 090601 (2004).

[11] M. Cencini, L. Palatella, S. Pigolotti, and A. Vulpiani, Phys. Rev. E 76, 051103.

[12] E. DelRe, B. Crosignani, P. Di Porto and S. Di Sabatino, Phys. Rev. E 84, 021112 (2011).

[13] R. Brito, M. J. Renne and C. Van den Broeck C, EPL 70, 29 (2005).

[14] P. I. Hurtado and S. Redner, Phys. Rev. E 73, 016137 (2006).

[15] G. Costantini, U. Marini Bettolo Marconi and A. Puglisi A, EPL 82, 50008 (2008).

[16] A. Fruleux, R. Kawai and K. Sekimoto, Phys. Rev. Lett. 108, 160601.

[17] A. Sarracino, A. Gnoli and A. Puglisi, Phys. Rev. E 87,
040101(R) (2013).

[18] T. G. Sano and H. Hayakawa, Phys. Rev. E 89, 032104 (2014).

[19] Dunkel J and Hilbert S, 2014 Nature Physics 10, 67.

[20] Huang K, Statistical Mechanics (J. Wiley, 1987)

[21] E. Paci and M. Marchi, J. Phys. Chem. 100, 4314 (1996).

[22] T. Tehver, F. Toigo, J. Koplik and J. R. Banavar, Phys. Rev. E 57, R17 (1998).

[23] D. Villamaina and E. Trizac, arXiv:1401.2683 (in press in EJP 2014).

[24] M. Smoluchowski, Ann. Phys. NY 21, 756 (1906).

[25] A. I. Khinchin, Mathematical foundations of statistical mechanics (Dover, 1949).

[26] D. Dürr, S. Goldstein and J. L. Lebowitz, Comm. Math. Phys. 78, 507 (1981).

[27] M. Costeniuc, R. S. Ellis, H. Touchette and B. Turkington, Phys. Rev. E 73, 026105 (2006). 